Ag shell was 1,600 ps. This radiative lifetime shortening is due to the Purcell effect resulting from plasmon whispering-gallery modes. $\mathrm{NH}$

\section{ROGUE WAVES}

\section{Surely deterministic}

Phys. Rev. Lett. 107, 053901 (2001)

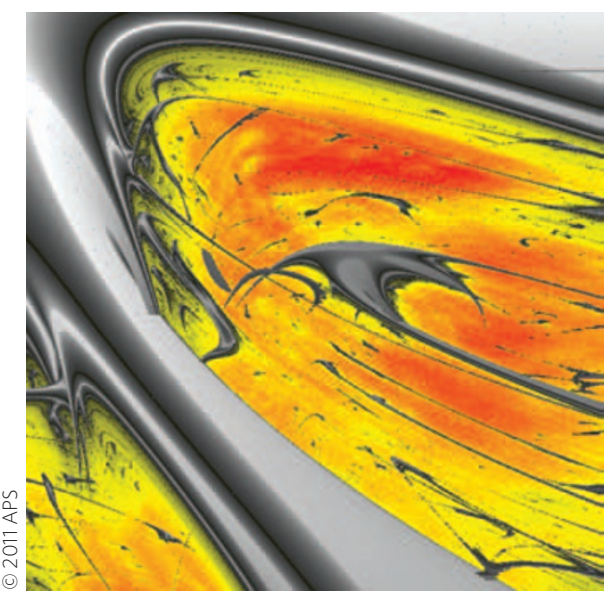

The physical mechanism behind the formation of rogue waves - waves with seemingly spontaneous large amplitudes has long been an interesting research topic for oceanologists and physicists, including those working in photonics. An important question is whether rogue waves can be described by a fully deterministic process with noise as a driving force. Cristian Bonatto and co-workers from Spain, France and Brazil recently carried out an investigation into the generation of rogue waves using a semiconductor laser that received optical injection from a continuous-wave master laser. The researchers not only showed that sporadic high-amplitude pulses can be observed with such a simple and inexpensive laser set-up, but also concluded that the rogue waves they observed are generated from deterministic nonlinearities. Their conclusion was based on good qualitative agreement between experimental results and simulated results from a simple, deterministic noise-free rate equation model.

\section{PHOTONIC CRYSTALS}

\section{Building LEDs}

Nature Mater. 10, 676-681 (2011)

Three-dimensional photonic crystals (3D PCs) are a promising platform for developing zero-threshold lasers, low-loss waveguides, high-efficiency LEDs and solar cells. However, the optoelectronic properties of 3D PCs are often unsuitable for practical applications because unoccupied chemical bonds at the device surface function as carrier traps, resulting in nonradiative recombination and consequently a significant decrease in device efficiency. Erik Nelson and co-workers from Kyoto University in Japan and the University of Illinois at Urbana-Champaign and the University of California at Santa Barbara in the USA have now used 3D-templatedirected epitaxial growth to fabricate a $3 \mathrm{D}$ PC LED. They fabricated a 3D structure with a lattice constant of $735 \mathrm{~nm}$ by filling a polymer template with alumina, removing the polymer and then epitaxially growing GaAs by metal-organic chemical vapour deposition. After fabricating the 3D framework, the researchers grew singlequantum-well GaAs/AlGaAs/GaAs as a passivation layer on the device surface. The 3D PC LED had a 15-nm-thick active layer of InGaAs and a cylindrical shape with a diameter of $120 \mu \mathrm{m}$. Electroluminescence spectra collected from the device showed a peak emission wavelength of $1,230 \mathrm{~nm}$ for drive currents of 2-8 mA.

\section{SENSING}

\section{Carbon ratio convenience}

Appl. Phys. B http://dx.doi.org/10.1007/ s00340-011-4666-1 (2011)

The ability to make precise measurements of the ratio between ${ }^{13} \mathrm{CO}_{2}$ and ${ }^{12} \mathrm{CO}_{2}$ in a gas sample is important for studying phenomena ranging from climate change and the release of volcanic gases to plant biochemistry and breath analysis. Although such measurements can be performed using isotope ratio mass spectroscopy, the necessary equipment is relatively large and complex, thus restricting it to laboratory use. Scientists from the University of Tokyo in Japan have now shown that wavelength modulation spectroscopy using a multipass gas cell and a $2 \mu \mathrm{m}$ semiconductor laser could help to enable the realization of mobile isotope ratio mass spectrometers. Their wavelength modulation spectroscopy set-up had a detection limit of 16 parts per billion, a response time of three minutes and good stabilization (standard deviation of $1 \sigma$ ) of $0.24 \%$ over 10 hours, which is sufficient for accurate and real-time measurements of atmospheric $\mathrm{CO}_{2}$. The researchers are considering the use of a double-cell system to further increase the precision of their measurements.

\section{SILICON PHOTONICS \\ Integrated isolators \\ Science 333, 729-733 (2011)}

The ability to confine the direction of light propagation - an important function for optical communications - is usually provided by optical isolators. However, these devices are often in bulk form and do not suit chip-scale integration. Liang Feng and co-workers from Caltech and the University of California at San Diego in the USA and Nanjing University in China have now designed and fabricated a waveguide system that performs one-wave photonic mode conversion at the telecommunications wavelength of $1.55 \mu \mathrm{m}$. By combining mode conversion with a mode filter, non-reciprocal light propagation can be achieved. The metallic-silicon waveguide ( $200 \mathrm{~nm}$ thick and $800 \mathrm{~nm}$ wide) is compatible with CMOS-semiconductor processing, thus paving the way for chip-based isolators. The researchers report that although their isolator has an insertion loss of around $7 \mathrm{~dB}$, this could be compensated for by incorporating optical gain into the device.

\section{BIOPHOTONICS}

\section{Subcellular sensing \\ Nano Lett. 11, 3431-3434 (2011)}

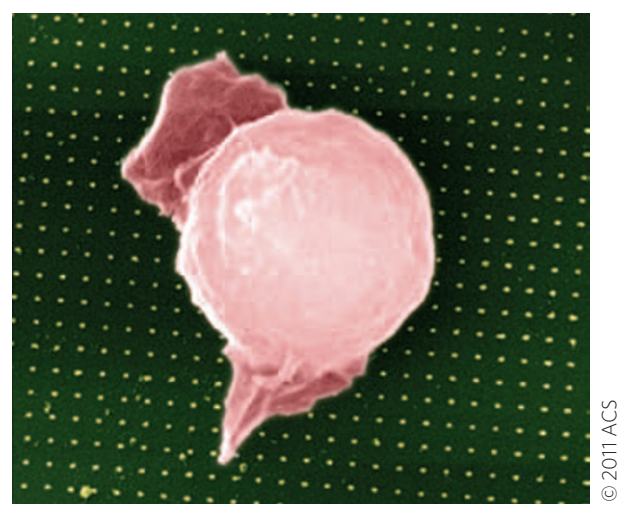

Cytokines are molecules secreted by cells for signalling and are essential to intercellular communication. Sheng Wang and colleagues from the University of California and Lawrence Berkeley National Laboratory in the USA have now developed a technique for imaging extracellular signalling at subcellular resolution. First, they used nanoimprint lithography to fabricate an array of plasmonic resonators, each $100 \mathrm{~nm}$ in diameter and comprising two $20 \mathrm{~nm}$ gold layers sandwiching a $5 \mathrm{~nm}$ silicon dioxide film, which together provided an optical resonance at $\sim 670 \mathrm{~nm}$. They then cultured the surface of the array with Jurkat T cells - a type of cell used to study T-cell leukaemia and T-cell signalling. They mapped interleukin-2 (IL-2, the cytokine signalling molecule) secreted by the cells using plasmonically enhanced fluorescence.

Written by Oliver Graydon, Noriaki Horiuchi, David Pile and Rachel Won. 